\title{
La pedagogía: Una mirada de estudiantes y profesores de programas de Licenciatura
}

Pedagogy: A Look of Students and Teachers of Bachelor Programs

Pedagogia: um olhar de alunos e professores de bacharelado

\author{
Yasaldez Eder Loaiza-Zuluaga* (ID) orcid.org/0000-0003-4215-2267 \\ Javier Taborda-Chaurra** (iD) orcidorg/0000-0001-5917-5778 \\ Franciso Javier Ruiz-Ortega*** (iD) orcid.org/0000-0003-1592-5535
}

Doi:

Para citar este artículo: Loaiza-Zuluaga, Y.E., Taborda-Chaurra, J. y Ruiz-Ortega, F.J. (2020). La pedagogía: Una mirada de estudiantes y profesores de programas de Licenciatura. Revista Colombiana de Educación, 1(79)., 13-38. https://doi.org/10.17227/rce.num79-8084

\begin{tabular}{lr} 
(C) $\left(\begin{array}{ll}\text { RC } \\
\text { (C) }\end{array}\right.$ & Evaluadocido: $12 / 06 / 2018$ \\
\hline
\end{tabular}

* Ph. D. en Ciencias de la Educación. Profesor titular del Departamento de Estudios Educativos y director del Doctorado en educación, Universidad de Caldas, Manizalez, Colombia. Correo electrónico: yasaldez@a ucaldas.edu.co

** Ph. D. en Ciencias de la Educación. Profesor titular del Departamento de Estudios Educativos, Universidad de Caldas, Manizales, Colombia. Correo electrónico: javier.taborda(a)ucaldas.edu.co

*** Ph. D. en Didáctica de las ciencias y las matemáticas. Profesor Departamento Estudios Educativos, Universidad de Caldas, Manizales, Colombia. Correo electrónico: francisco.ruiz@ucaldas.edu.co 


\section{Resumen}

El artículo es resultado parcial de la investigación "Semiosis de la Pedagogía, el Currículo y la Didáctica" y se focaliza en la categoría pedagogía. Es un estudio cualitativo que tiene como objetivos describir y valorar las ideas que en torno al campo de la pedagogía manifiestan profesores y estudiantes de la Universidad de Caldas, Colombia. En lo metodológico tuvo en cuenta una muestra intencional de 168 sujetos que aplicaron a un instrumento de 39 ítems de los cuales 11 aludían a asuntos relacionados con la pedagogía. El análisis se hizo a partir de las valoraciones dadas a los diferentes ítems utilizando herramientas de Excel. Se destacan en los resultados valoraciones importantes que se hacen de la pedagogía como arte o como campo de conocimiento y de la experiencia como un componente central en la constitución de la formación y del conocimiento pedagógico de los docentes.

\section{Palabras clave}

pedagogía; estudiantes; profesores; formación; educación; experiencia

\section{Keywords}

pedagogy; students; teachers; training; education; experience
Abstract

The article is part of the research "Semiosis of Pedagogy, Curriculum and Teaching" and focuses on the category pedagogy. This qualitative study aims to describe and assess ideas about the field of pedagogy of teachers and students from the University of Caldas, Colombia. Methodologically, it considered a purposive sample of 168 subjects who applied to an instrument of 39 items of which 11 issues alluded to pedagogy The analysis was based on the scores given to the different items using Excel tools. The results highlight the understandings of pedagogy as art or field of knowledge, and of experience as a key element in the constitution of formation and pedagogical knowledge of teachers.

\section{Resumo}

O artigo é resultado parcial da pesquisa "Semiose de Pedagogia, Currículo e Didática" e concentra-se na categoria de pedagogia. Trata-se de um estudo qualitativo que tem como objetivo descrever e valorizar as idéias que professores e alunos da Universidade de Caldas, Colômbia, expressam sobre o campo da pedagogia. Metodologicamente, levou-se em consideração uma amostra intencional de 168 sujeitos que se inscreveram em um instrumento de 39 itens, dos quais 11 aludiram a questões relacionadas à pedagogia. A análise foi realizada a partir das avaliações realizadas nos diferentes itens, utilizando as ferramentas do Excel. Importantes avaliações da pedagogia como arte ou como campo do conhecimento e da experiência como componente central na constituição da formação de professores e do conhecimento pedagógico são destacadas nos resultados.

\section{Palavras-chave}

pedagogía; estudantes; profesores; formação; eđucação; experiencia 


\section{Introducción}

La preocupación por alcanzar mayores comprensiones en torno a las relaciones entre pedagogía, currículo y didáctica en el contexto institucional, centra el interés en este artículo; no obstante, de ese amplio escenario de estudio, nos ocuparemos fundamentalmente de lo que los estudiantes -que realizan su práctica docente de diversos programas de formación de educadores-y sus maestros conciben de la pedagogía, de cómo se constituye conocimiento pedagógico y del papel que desempeña la experiencia de los profesores en la constitución de este.

\section{Educación y formación: consideraciones básicas}

Reflexionar y repensar sobre conceptos de educación y formación es uno de los retos en los programas de formación docente, como mecanismo de comprensión de su pensamiento y desempeño en el aula. Ello seguramente permitiría diseñar estrategias eficientes y eficaces para responder a "requerimientos de la educación actual y de las pedagogías contemporáneas" (Loaiza, 2018, p. 7).

Ahora bien, en el campo de la educación, es imperativo tener presente que en la modernidad temprana empezó a constituirse una manera de decir de algo que, expuesto como acontecimiento a los hombres, requería ser bien nombrado. Ese fenómeno al cual hacemos alusión es educación. Como fenómeno vivo se configura de manera especial y es objeto de orientación intencional e institucionalizada, según intereses muy disímiles. Por lo anterior, a este fenómeno se le menciona como social y cultural, por cuanto no depende solo de disposiciones biológicas de los humanos sino que obedece, como lo plantearía Durkheim (1976), a voluntades de generaciones adultas que en nombre de cierta experiencia asumen como tarea reproducir ${ }^{1}$ en otros los bienes más preciados de las organizaciones humanas, del arte y la cultura y, en otros casos, de hacer explícitos los fundamentos de la convivencia social y de los valores que las nuevas generaciones deberán interiorizar y compartir. En una u otra perspectiva se ha definido el rumbo de la educación; en otras palabras, desde una perspectiva reproductora del orden y de las ideas, o una que deja abierta una nueva constitución de sujetos, en cuanto se dejan claros los principios pero no todo el trazado de la humanidad querida. ${ }^{2}$

1 Según Bourdieu y Passeron (1970), la educación es el agente fundamental de reproducción, de la estructura de las relaciones de poder y de las relaciones simbólicas entre las clases; los autores ponen énfasis en la importancia del capital cultural heredado en la familia como clave del éxito en la escuela.

2 Cerleti (2016) configura apreciaciones cercanas a las expuestas cuando apunta que: "asumiremos, entonces, como punto de partida, que cada acto educativo actualiza un 
Vamos a apoyarnos inicialmente en Benner (1991). Para él, las nociones de educación afirmativa y no afirmativa aluden a discursos sobre la educación y a prácticas sobre la educación que van en la dirección, respectivamente, de lo institucionalizado y prescrito o de lo incierto, no institucionalizado, azaroso y no prescrito. Educación afirmativa y no afirmativa confirman intencionalidades completamente diferentes; de un lado, una que intenta someter y direccionar absolutamente todo en la formación (o deformación) de los sujetos; de otro lado, aquella que le apunta a abrir espacios de libertad, de participación, de asombro a los sujetos.

En este sentido, se sabe que en la abundante literatura que la modernidad temprana y tardía ha producido, el asunto de la educación intencional ${ }^{3}$ y no intencional ha tenido tratamiento a niveles distintos con el asunto de la formación. En cuanto acto intencional, es evidente que la educación, según lo expuesto por Trujillo (2006),

[...] es una actividad cuyo abordaje teórico está cargado de conceptos de carácter teleológico, de fines, de metas. Asumir que la educación cumple una función social implica apostar por una posición en cuanto a qué es lo que se enseña y como se enseña. (p. 97)

Esto supone una carga de intencionalidad formativa de los sujetos que enseñan sobre los que aprenden.

De otra parte, en la relación que puede darse entre educación y formación, es evidente que una u otra aparecen, según sea el caso, como hiper- o hiponímicos, dependiendo de si la formación se entiende como sinónima de educación, o si la formación es declarada explícitamente como finalidad de aquella. De nuestra parte, esta discusión puede resultar estéril, por cuanto se han venido constituyendo ambas como campos de saber de tal importancia que, por ejemplo, a la disciplina que se encarga específicamente de la formación se le ha ido configurando un campo especializado al que se le denomina formática (Honoré, 1980); y en el caso de la educación, al campo conocido como pedagogía.

Vista como finalidad de la educación o como sinónimo de esta, hay asuntos que a la formación confluyen y los cuales es necesario mencionar. En primer lugar, debe destacarse que al respecto hay cuestiones que lo matizan. Una de ellas hace referencia a que al hablar de formar hacemos

problema filosófico y político fundamental, que es cómo resolver la tensión entre reproducir lo que hay y dar lugar a lo diferente que puede haber. La historia de la educación podría leerse a la luz de mayor o menor peso que se ha intentado dar a una u otra de estas alternativas" (pp. 16-17).

3 García (1986, p. 133) plantea la necesidad de "clarificar el significado que usualmente se le concede a los términos de 'intención', 'intencionalidad', 'educación intencional' e 'intención educativa' en el discurso pedagógico"; identificando que no se evidencia una "adecuada integración en el discurso pedagógico de dichos conceptos", aspecto que, considera, puede darse en virtud de la polisemia de los términos. 
alusión a una intencionalidad de alguien (una persona o una institución) que establece una relación con un sujeto que ha de considerarse sujeto de formación, esto es, de sujeto a formar. Tal relación es en parte de ayuda, de formación, en la cual, en una perspectiva muy tradicional, se puede definir como formar al otro, como moldearlo y definirlo (muy propio de la educación afirmativa) o en otra dirección, como acompañar, cultivar las capacidades y las potencialidades del otro. En tal proceso asociado al formar, puede darse en la dicotomía moldeamiento o cultivo de múltiples variantes.

En segundo lugar, hay un matiz especial que fue ampliamente desarrollado en la primera modernidad y que encuentra soporte en el ciclo del Meister de Goethe $(1968,2008)$ pero que ha ido cobrando nuevas nominaciones de la mano de progresos en comprensiones del fenómeno educativo, y que acompaña al desarrollo de la autonomía de los sujetos. Formarse es la cuestión anunciada; y cuando se habla de ella se hace referencia a la capacidad de cada sujeto de dirigir de manera responsable su propia constitución como humano; se hace mención a la formación de sí, al cultivo de sí, en fin, a la autoformación.

El tercer matiz nos trae al asunto de la formación. Esta, según Gadamer (2005), alude a uno de los cuatro conceptos básicos del humanismo al lado del juicio, el sensus communis y el gusto. A la formación la reclama el autor vinculada a la cultura y a la constitución de la humanidad. Hace referencia a ella criticando primero la intención de reducirla a moldeamiento externo y pasa a enunciarla como un asunto en el cual la relación de ayuda en la constitución del otro en relación con otros es fundamental. Son para Gadamer (2005, p. 38) categorías asociadas a la formación así descrita, el aprendizaje, la enseñanza y la competencia personal.

Complementa los planteamientos que se han venido haciendo lo expuesto por Sánchez, Ramírez, Chica y Ávila (2012). Para ellos la educación "es un proceso que se funda y desenvuelve a partir de la capacidad inmanente del ser humano de desplegar todas sus capacidades y potencialidades y de la sociedad de estar en permanente cambio y transformación" (p. 38). En lo que plantean, los asuntos de la educación y de la formación como devenir están presentes; la formación como asociada a una cierta disponibilidad, al despliegue de algo que nos caracteriza como especie relacionado con una capacidad que puede ir más allá hasta constituirse en habilidad y posteriormente en experticia, en tanto somos a la vez potencia. La educación en esta perspectiva no está fundada para siempre.

Sánchez et ál. (2012), además, manifiestan que la educación "es una práctica que se mueve en la tensión entre la intencionalidad y la no intencionalidad de los sujetos y grupos sociales que en ella intervienen" (p. 39). Asunto que nos Ilevaría a plantear que no siempre los procesos educativos que se desarrollan están mediados por toma de conciencia conceptualizada del asunto, lo que podría ser bien visto en sujetos no

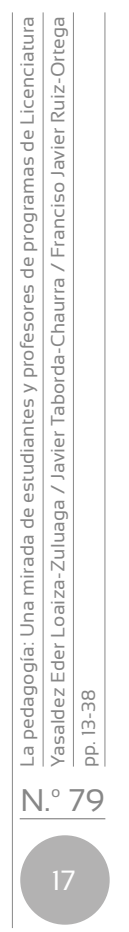


formados como para desempeñarse en labores docentes, pero que sí podría considerarse una falencia en cuanto, para la constitución de conocimiento pedagógico, la reflexión en la acción de educar es fundamental.

\section{La experiencia en la constitución de conocimiento pedagógico}

La experiencia, según la tradición de ideas que la soporte, adquiere diversa connotación. Nos alejamos de considerarla solamente como experimento, como conjunción solidaria de hipótesis a probar con asuntos perfectamente definidos y estructurados a partir de los cuales unos determinados resultados han de derivar. Iluminemos lo dicho con consideraciones más precisas en torno a la experiencia.

Bollnow (2001) dice de la experiencia que ella provoca cambios permanentes en quien la vive; entonces, constituye un asunto del que se puede decir que se extrae "una enseñanza general" (p. 145). La experiencia deviene de lo que se nos viene, o sea, de algo fortuito "ajeno a cualquier propósito". En la misma perspectiva se manifiesta Jay (2003): "la experiencia tiene que ver con algo que sucede cuando uno no lo espera, cuando uno no lo planifica, cuando uno se ve sorprendido por los hechos" (p. 22), lo cual no implica, desde nuestra perspectiva, que sobre esta experiencia los sujetos no puedan conversar.

Larrosa (2006), al hacer alusión a la experiencia, asume para su análisis la frase: "eso que me pasa" (p. 44). En tal enunciado, "eso" hace referencia a algo imprevisto. "Eso", en el aula, es lo incierto, así intencionalmente hayamos definido ambientes y contenidos para promover aprendizajes. Eso, de "eso que me pasa" en el aula, se constituye en experiencia significativa, en experiencia monumental, en experiencia cumbre, en la medida en que se viene hacia mí sin previo aviso.

En "eso que me pasa" el "me" es un reflectivo que da cuenta de que eso que pasa me pasa a mí de una determinada manera y no a otro. En tal sentido, lo que pasa en el aula no le pasa igual, no afecta igual a dos maestros distintos. La experiencia sería así idiosincrática y respondería a lo que coloquialmente podemos mencionar con la mediación de la frase "nadie experimenta por cabeza ajena". En "eso que me pasa", de otra parte, lo que pasa puede constituir acontecimiento no reflexionado o constituirse en asunto del pensamiento, por cuanto el que experimenta lo asume como objeto de reflexión.

De otra parte, es necesario asumir que la experiencia de los docentes en el aula se demuestra en sus prácticas pedagógicas y didácticas, para lo cual vale la pena retomar lo planteado por Castañeda (2018, p. 14), quien expone que 
[...] la práctica pedagógica es el momento en el cual los profesores despliegan sus conocimientos en un aula de clase en vivo y allí ponen en juicio sus criterios pedagógicos para realizar actos de educación (Richards y Nunan, 1990; Woodward, 1992). Ese momento, el de la práctica, puede ser un sitio epistemológico rico en información para que los programas de formación de docentes (re)piensen su espíritu formativo a partir de las experiencias vividas por los practicantes.

Esta postura, permite comprender, entonces, que es en la puesta en escena de los conocimientos y la formación adquirida, que se da fe de la experiencia adquirida y del impacto de esta en el desempeño de quienes se dedican a la labor magisterial; aspecto que denota la importancia de reflexionar sobre dichas prácticas y sus posibilidades de mejora.

Al acto de reflexión se apegan varios autores para establecer diferencia, precisamente, entre la experiencia ingenua y la experiencia reflexionada (Larrosa, 2006; Dewey, 2008). La experiencia ingenua es aquella asociada al acontecimiento, a sucesos del día a día que poco efecto tendrán en las consideraciones del sujeto. La reflexionada es la experiencia mayor, aquella que asociada a "lo que me pasa" deja huella en el sujeto por cuanto, con la mediación de la reflexión, se constituye ella en suceso cumbre, en experiencia monumental.

En relación con la experiencia, otras consideraciones suelen aparecer. Por ejemplo, en Bollnow (2001) hay algo de doloroso en muchas experiencias. En Csikszentmihalyi (2008), las experiencias óptimas pueden gozar, entre sus características, de un nivel alto de disfrute por parte de los que las viven. En cualquiera de los dos casos aparece como común a la experiencia el que aquella que complejiza el ser es la que se constituye en objeto de atención y reflexión por parte de los que viven experiencias.

De lo dicho llama la atención que la experiencia que interesa en la educación institucionalizada y en pedagogía es aquella experiencia reflexionada; que en situaciones disímiles, previsibles en parte o no, complejizan la personalidad del docente y del alumno; aportan a la reflexión pedagógica mucho más de lo que la misma pedagogía es al momento para el docente; esto es, transforma al sujeto, a los sujetos, al maestro, al estudiante y a la misma pedagogía, yendo más allá de ella al superarla. Ya no hablaríamos de una pedagogía de la caverna sino de una que va en pos de mayor vitalidad; una pedagogía del riesgo, en la perspectiva en que nos la muestra Freire y Faundez (2013) asociada a la pregunta más que a respuestas.

Con lo dicho se hace énfasis en la pertinencia de la categoría experiencia para tender puente entre pedagogía y formación. Pero, más allá, debemos reconocer en ella un asunto sustancial en la constitución de la pedagogía; como lo plantean en la dirección predicha Cruz y Taborda 
(2014), la pedagogía es teoría de la experiencia del educar y tiene como valor adicional el que puede ser "comunitarizada": se puede hablar a otros sobre ella.

\section{La formación de educadores}

Las instituciones formadoras de educadores en el mundo siempre han luchado por ser consideradas escuelas profesionales con independencia, con una vida y un fin propios. Así mismo, en diversos países han sufrido sus respectivos avatares según la política dominante la cual hace que su problemática sea orientada hacia la política del Estado, influyendo lógicamente en la enseñanza en general, condicionando, de alguna manera, los componentes del modelo educativo que orienta la formación de los educadores.

Ahora bien, reflexionar en la formación de educadores, invita a pensar en cómo se ha dado este proceso a través de la historia; en virtud de ello, es necesario reconocer que el modelo más antiguo de formación de profesores ${ }^{4}$ se sustentó en la observación y la emulación de un maestro, situación que "ha fragmentado los saberes y ubicado la formación en un escenario desprovisto de reflexiones profundas e integrales sobre los saberes, el currículo, la enseñanza y el aprendizaje" (Ruiz, Márquez, Badillo y Rodas, 2018, p. 560). Considerar, entonces, la variedad histórica de la formación de los educadores, desde un planteamiento pedagógico triplemente desplegado, se puede remontar a tres grandes modelos que, a su vez, corresponden a tres antropologías claramente diferenciables. Según expresa Parnett (1986, p. 50), retomando los postulados de Pestalozzi, el hombre puede ser considerado como obra de la naturaleza, de la sociedad o de sí mismo y, de manera correspondiente, al educador se le define, respectivamente, como auxiliar de la naturaleza, agente de la sociedad o representante de una humanidad realizada, es decir, como persona. ${ }^{5}$

4 Mucho antes de que existieran escuelas especiales para el entrenamiento de profesores, ya se contaba con recomendaciones y métodos de instrucción escritos para los profesores. Los profesores romanos pudieron leer las recomendaciones de Quintiliano sobre enseñanza; los del siglo xvı aprendieron de los escritos de Erasmo; y los profesores del siglo xvı pudieron leer la Didáctica Magna, en la que Comenio expone que el principal objetivo era encontrar un método de instrucción mediante el cual los profesores probablemente enseñaran menos pero los alumnos aprendieran más. (Véase Carmona y Jacobs, 1975)

5 Esta expresión, al tiempo, deviene como herencia de planteamientos de Rousseau quien, en los prolegómenos del libro primero de su obra Emilio o de la educación, manifiesta: "La educación es efecto de la Naturaleza, de los hombres, o de las cosas. La de la Naturaleza es el desarrollo interno de nuestras facultades y nuestros órganos; la educación de los hombres es el uso que nos enseñan estos a hacer de este desarrollo; y lo que nuestra experiencia propia nos da a conocer acerca de los objetos cuya impresión recibimos, es la educación de las cosas" (Rousseau, 2005, p. 2). 
Para el caso de Colombia, en el contexto educativo las instituciones formadoras de maestros, se resaltan tres elementos que determinan la historia de la educación pública y, que de una u otra manera, han incidido en el surgimiento de reformas educativas dirigidas a las entidades formadoras de educadores; sea del caso mencionar entonces que, en el desarrollo e institucionalización de dichas instituciones se han vivido las siguientes etapas: a) la pérdida de legitimidad de la Iglesia católica que dominó la orientación de la enseñanza en este país desde la Colonia y se extendió a lo largo de casi todo el siglo XIX, pero que tomó forma a partir de la Constitución de 1886, en la cual se llegó a una transición, en la que el Estado conservaba la inspección, pero la Iglesia definía los contenidos de la educación; b) la influencia de las misiones pedagógicas, especialmente las alemanas y la injerencia determinante de los organismos internacionales controlados por Estados Unidos en la década de 1950, factor que condiciona los aspectos fundamentales del devenir histórico de la educación colombiana desde entonces; y c) la lucha de resistencia librada por el movimiento magisterial, por los estudiantes, por la comunidad educativa y por la sociedad misma en defensa de la educación pública6 ${ }^{6}$.

Fruto de la lucha magisterial se presenta en Colombia el Movimiento Pedagógico $\mathrm{Nacional}^{7}$ del cual surge y se aprueba la Ley General de Educación (Ley 115 de 1994) con la cual se viste de autonomía a las instituciones educativas, a partir de la elaboración de los proyectos educativos institucionales (PEI); posteriormente a esta legislación aparece el Decreto 3012 de 1997 (referido a las Escuelas Normales y su acreditación como instituciones formadoras de educadores) y el Decreto 272 de 1998 -derogado por el Decreto 2566 de 2003-, con el cual se estipulaban las condiciones mínimas de calidad para los programas de licenciatura y especializaciones en educación y se establecía como disciplina fundante de la formación de educadores la pedagogía; con la intención seguramente de intentar darle identidad a la profesión docente desde una disciplina que cimente su desempeño.

6 Recuérdese que la Federación Colombiana de Educadores se fundó en 1959, y que desde esa época se han iniciado las más fervientes luchas del sector educativo. De otra parte hay que destacar el impacto que generó el movimiento estudiantil de 1971, el cual logró la participación de la comunidad educativa en general en diversos sectores del país. (Véase Ocampo, 1999).

7 Periodo circunscrito desde 1982 por una corriente de pensamiento que oficialmente se denominó Movimiento Pedagógico, que surgió del XII Congreso de la Federación Colombiana de Educadores (Fecode), realizado en Bucaramanga; hasta, 1994 con la promulgación de la Ley General de Educación 115 del 8 de febrero del mismo año. (Véase Loaiza, Pineda y Arbeláez, 2014, p. 63). 
Es claro que la formación de educadores en Colombia ha pasado por varias reformas e implicaciones que han afectado, de alguna manera, la forma como se ha concebido el rol y la formación docente; por ello, en la actualidad, pensar en la formación de maestros en Colombia implica pensar en la necesidad de una política integral que piense todas las dimensiones que afectan y potencian esta labor; en tal sentido, la formación de educadores, como se plantea en el Sistema colombiano de formación de educadores y lineamientos de política (MEN, 2014); debe pensarse

[...] desde un referente conceptual que admita su magnitud y dimensiones, más allá de una definición de condiciones de entrada, de la administración de mecanismos o herramientas de actuación profesional y de la verificación de su adquisición y adecuado uso; además de tal propósito de cualificar la labor docente, se deben generar propuestas alternativas metodológicas y organizacionales que propicien la consolidación, diseño, aplicación y sistematización de experiencias innovadoras, soportadas desde el rigor académico, investigativo y curricular, en coherencia con las necesidades actuales de la educación colombiana y con la intención además, de dar respuesta con una clara intención de generar liderazgo local, regional y nacional, en el plano educativo, investigativo y creativo. (p. 46)

En virtud de lo anterior, es válido también retomar los planteamientos que se dieron en el marco de la XVII Conferencia Iberoamericana de Ministros de Educación, realizada en El Salvador (OEI, 2008), en la cual se planteó que para mejorar la formación del profesorado se requiere de

[...] un profesor que cuente con una formación inicial de calidad y con las oportunidades de acceder a programas de capacitación continua, para que pueda contribuir al mejoramiento de los resultados de los niños en su rendimiento. Sin embargo, en la mayor parte de los países de la región, las inmensas demandas de transformación pedagógica que se les han exigido a los docentes en las últimas décadas no han sido acompañadas de los debidos procesos de cambio de las instituciones que los forman, ni de las condiciones de trabajo y de desarrollo profesional necesarias. (p. 16)

En tal sentido, es necesario pensar que la formación de los educadores debe ubicarse en una nueva dinámica en la que el docente en formación y en ejercicio recapacite su acción desde la reflexión de su práctica y las posibilidades de contribuir a una educación de mayor calidad ${ }^{8}$; de tal suerte que asuma una postura diferente a la que tradicionalmente ha

8 "...como ha señalado la Unesco en diversas ocasiones, los profesores no son el problema, sino la solución. Efectivamente, los profesores son el factor clave de la calidad de la educación. En Europa, nunca ha tenido éxito una reforma educativa que se haya querido llevar a cabo sin contar con la colaboración y la aceptación del profesorado" (Prats y Raventós, 2005, p. 20). 
soportado su desempeño, la cual se ha fundamentado en la transmisión de información y contenidos a los alumnos, en tanto las exigencias actuales conllevan a que el educador asuma una postura crítica y contextualizada que le permita, primero, enfrentar la educación de hoy y del futuro a partir de formas de trabajo centradas en la reflexión permanente de sus prácticas pedagógicas, y segundo, generar saber pedagógico 9 .

Este tipo de conocimiento en la práctica pedagógica significa, desde el análisis de Pozo, et ál. (2006), sobre la propuesta de Schön, que:

El docente puede reflexionar en medio de la acción sin necesidad de interrumpirla. Una sorpresa en la dinámica del aula, una variación inesperada en la aplicación de una rutina, suscitaría un proceso de reflexión dentro de una acción-presente que el autor considera que resulta en alguna medida consciente, aunque no se produzca necesariamente por medio de palabras. Tenemos en cuenta el conocimiento inesperado y el conocimiento en la acción. Nuestro pensamiento se vuelve, pues, sobre el fenómeno y, a la vez, sobre sí mismo. Lo que distingue la reflexión en la acción de otros tipos de reflexión es su inmediata relevancia para la acción, tanto para la presente como quizás otras que consideremos similares. Un buen profesor mostraría una alta capacidad para integrar la reflexión en la acción en una tranquila ejecución de su tarea. (p. 84)

En tal sentido y apoyados en Schön (1987), el conocimiento que surge de la reflexión en la acción y posterior a la acción es un nuevo conocimiento, un conocimiento práctico. Este es generalmente tácito y el profesor debe hacerlo explícito para generar transformaciones. El conocimiento que se constituye a partir de la reflexión en la perspectiva de Schön tiene su análogo, en la perspectiva que describimos en el apartado anterior, en la experiencia reflexionada, en la experiencia vinculada al acto de educar que, como se expuso, ayuda a la constitución del conocimiento pedagógico en cuanto, también, puede ser comunitarizada. De ninguna manera se niega en lo expuesto la importancia de los Ilamados saberes constituidos; se llama la atención más bien en torno a la necesaria articulación entre estos y los denominados saberes de la experiencia (Cifali, 2005, p. 179).

De otra parte, también es necesario comprender que, como plantea Angulo (2012), "el ejercicio de la docencia constituye una actividad intencional, de allí que se entiende desde el sentido que le dan los educadores a lo que hacen y a lo que se proponen conseguir en su quehacer educativo" (p. 12). En ese sentido, retomando los planteamientos de Contreras (2001), según la intencionalidad del maestro, se pueden distinguir tres miradas

9 Los docentes, dice Díaz (2006), "generan teorías de manera consciente o inconsciente, que pueden contribuir a la construcción de una base de conocimientos sobre los procesos que explican su actuación profesional y el reconocimiento de esta realidad constituye un nuevo referente para analizar la formación permanente del docente" (p. 88).

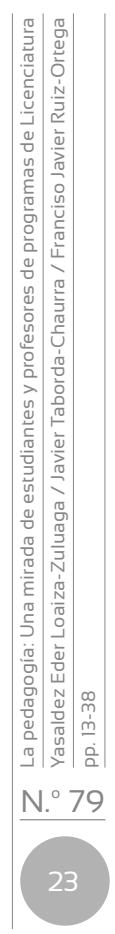


diferentes del ser educador: el experto técnico, el reflexivo y el intelectual crítico. He allí ya un asunto que ameritaría un análisis en profundidad sobre cuáles son los modelos que han asumido las instituciones dedicadas a la formación de educadores ${ }^{10}$.

\section{Metodología}

La investigación es un estudio de caso desarrollado en la Universidad de Caldas y forma parte de una investigación más amplia titulada "Semiosis de la pedagogía, el currículo y la didáctica", de la cual, en la presente entrega, se dedica atención especial a la categoría pedagogía.

La investigación es cualitativa y de ella, en esta ocasión, se presentan los resultados obtenidos sobre el pensamiento de docentes y estudiantes de los programas de formación de educadores, en relación con el asunto de la pedagogía. El instrumento utilizado para la recolección de información fue un cuestionario de 39 items, de los cuales 11 estaban dedicados a recoger información sobre pedagogía. Estos presentaban una proposición para que los estudiantes y sus maestros dieran una valoración del grado de aceptación de esta. Tal valoración está asociada a una escala de Lickert modificada en la que, sobre una línea de diez centímetros que iba de la sigla CA (completo acuerdo) a CD (completo desacuerdo), los consultados señalar con una equis su grado de preferencia.

La muestra de estudiantes fue intencional. En relación con estos, se aplicó como único criterio de selección que estuviesen realizando la práctica educativa, fase ubicada en el último año de la formación de docentes en los diferentes programas de licenciatura de la universidad. El total de estudiantes fue de 156. En cuanto a los docentes, se tuvieron dos grupos: el primero conformado por 8 coordinadores de la práctica educativa de las licenciaturas de la universidad (7 coordinadores de las diferentes licenciaturas, más la coordinadora general de la práctica) y, el segundo, integrado por 4 docentes elegidos al azar.

10 Lo anterior invita a pensar que "la formación docente entonces no puede ser una mera revisión de fórmulas didácticas o un adiestramiento en disciplinas específicas, tiene que ser el espacio que acoja la inquietud del profesor por trascender, el lugar en donde, mediante la reflexión, pueda aclarar su posición respecto de la problemática educativa, su rol en la dinámica social, su forma de entender el mundo" (Bustamante, 2006, p. 44). 
La investigación responde a preguntas que tienen que ver con el cómo los maestros y los estudiantes practicantes asumen el asunto de la pedagogía, cómo consideran que se construye conocimiento pedagógico y qué papel consideran que cumple la experiencia en la constitución de tal saber. El análisis que se hace de las valoraciones dadas por docentes y estudiantes a las proposiciones planteadas en el instrumento y que indagaban sobre las cuestiones expuestas, tuvo como procesos complementarios para su discusión, la descripción, desde la elaboración de gráficas que muestran el valor asignado a cada una de las proposiciones y la interpretación, desde la discusión de los resultados a la luz de referentes teóricos significativos en el campo de la pedagogía.

El diseño de la investigación contempló inicialmente la configuración de un referente conceptual que permitiese la posterior interpretación de los resultados. Implicó, en un paso siguiente, focalizar atención en ítems del instrumento general que sirviesen a la pesquisa. De la información general recolectada para el macroproyecto se seleccionaron las preguntas que hacían referencia a la pedagogía. En la etapa de análisis se aprovecharon herramientas de Excel para ingresar, codificar, procesar y graficar la información. Luego, se procedió a la fase de interpretación y discusión de lo cual derivan algunas conclusiones.

\section{Resultados y discusión}

\section{Valoración comparada y general de la categoría pedagogía}

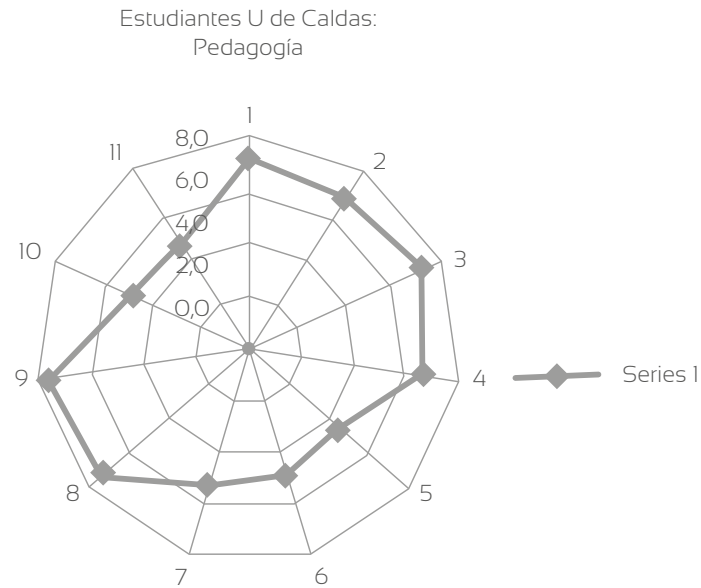

Figura 1. Valor que dan a la pedagogía estudiantes de licenciaturas en Educación de la U. de Caldas

Fuente: elaboración propia. 


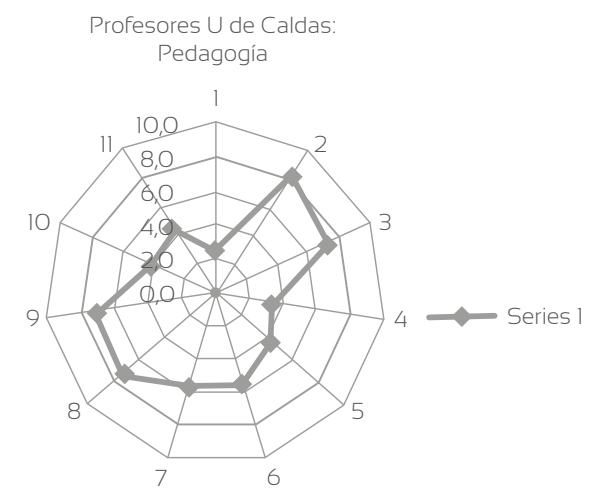

Figura 2. Valor que dan a la pedagogía profesores de licenciaturas en Educación de la U. de Caldas

Fuente: elaboración propia

Las figuras 1 y 2 representan cómo valoran la pedagogía estudiantes y profesores de la Universidad de Caldas. En el exterior, numerados de 1 a 11, aparecen los ítems y en el tejido, de adentro hacia fuera, de 0 a 10, el valor que a cada uno de ellos otorgaron los sujetos.

A primera vista, hay una notoria diferencia en la tendencia de las gráficas de los estudiantes con la de los profesores. Sin embargo, específicamente, cuando se revisan los ítems y resultados ¿a qué podemos estar haciendo referencia?

El ítem 1 consultaba el grado de acuerdo con la expresión "La pedagogía es el arte de enseñar", y el ítem 2 el grado de acuerdo con "El objeto de estudio de la pedagogía es la formación humana". Sobre el primero parece haber acuerdo entre los estudiantes en asociar con una valoración muy alta el asunto de la pedagogía con la enseñanza. Contrasta lo dicho con la valoración de los maestros de la Universidad de Caldas, para quienes la pedagogía, al parecer, no es el arte de enseñar. Si se observa el ítem 2, hay mucha cercanía en la valoración de los estudiantes y de los profesores, en cuanto consideran que el objeto de la pedagogía es la formación humana.

Los resultados que dan gran valor a la pedagogía como arte de enseñar, hasta el momento podrían deberse a que los estudiantes realizan, casi con dedicación exclusiva, su práctica profesional y están dando gran importancia al hacer; al parecer, aún no amplían su marco de referencia para empezar a comprender la pedagogía más allá de tal quehacer instrumental, cuestión que en los maestros sí podría estar influyendo. En cuanto a la coincidencia en el asunto de la formación en estudiantes y profesores, es posible que los discursos en la Universidad de Caldas como institución educativa de educación superior se estén centrando en asumir esta categoría como finalidad de la educación y como núcleo central de las discusiones de los pedagogos. 
Ahora bien, optar por describir la pedagogía como arte de enseñar ha permitido colocar en primer plano un asunto que varios autores han señalado en relación con los enseñantes. Quizá fue inicialmente Aracelly de Tezanos (2006) quien en Colombia colocó de relieve el traslapamiento de la pedagogía por la didáctica. A pesar de declarar los maestros la importancia de la pedagogía, al momento de hacer enunciados o declaraciones sobre ella daban cuenta de asuntos relacionados con el qué hacer y cómo hacerlo, razón por la cual metafóricamente llama a los maestros artesanos intelectuales, en tanto intelectualizamos lo que hacemos y tal intelectualización, que podría ser más propia de la didáctica, la asumimos como pedagogía. Loaiza, et ál. (2008) también revisan tal traslapamiento en maestros de una institución educativa del sur de Colombia. Para las autoras, la enseñanza y algunas declaraciones sobre ella son denominadas por las maestras y maestros como pedagogía. Igual idea soporta Taborda (2009) a partir de reciente indagación.

Sobre la formación, la coincidencia casi plena en declararla objeto de la pedagogía se asocia a múltiples discursos devenidos de perspectivas educativas de la ilustración en cuanto ella se anunciaba como finalidad de la educación y en la cual las escuelas o instituciones de educación podían influir (véase, Kant, 1983; Hegel, 2000; Goethe, 1968, 2008; Schiller, 1932; entre otros). Ya el asunto del conocimiento como propio de una casta o clase, con estos autores, se había superado. Por algo empezaba a decirse que el hombre es lo que es por su educación; es decir, la formación de los sujetos dice mucho de los procesos educativos por los cuales el mismo ha pasado.

Es claro que en el tercer ítem, en el que se consultaba acerca de si la pedagogía orientaba el acto de enseñar en el aula, las valoraciones fueron altas de parte de estudiantes y profesores. La pedagogía hace que lo que se realice en el aula tenga sentido en el trayecto de la formación de los estudiantes. Ahora bien, cobra sentido en dimensión diferente para estudiantes y profesores. Si se revisa la valoración que se da al ítem 4, "La pedagogía es indispensable para el moldeamiento de los alumnos en clase", se encuentra gran contraste. Los alumnos de la Universidad de Caldas le dan gran valor a este ítem; para ellos la pedagogía sirve al moldeamiento enunciado. Para los profesores la valoración es mucho más baja, algunos al parecer están de acuerdo con este planteamiento, pero en promedio, el enunciado tiene baja valoración. Podría decirse, vinculando los ítems 3 y 4, que la pedagogía en el caso de los estudiantes orienta el acto de enseñar, pues les ayuda al moldeamiento de los alumnos. Esto podría explicarse porque en los estudiantes de práctica, novatos en el asunto de enseñar, una de sus mayores preocupaciones es el control de los alumnos, cuestión que, por su extendida práctica profesional, puede ser relativizada por los profesores universitarios.

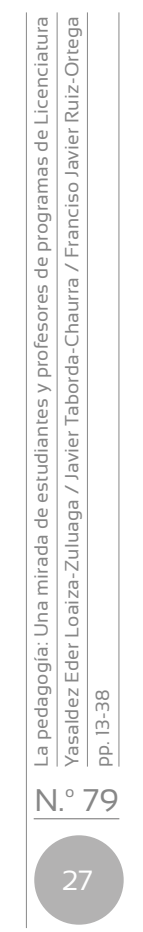


Los acuerdos sobre la formación y el moldeamiento de los estudiantes nos recuerdan los planteamientos de Foucault (2005) en relación con las funciones de instituciones como la escuela. Hechas al parecer para vigilar y castigar, no guardan mucha diferencia con las valoraciones que hacen de ella nuestros encuestados. Al estar de acuerdo con la formación o con la educación como objeto de estudio de la pedagogía, esta representaría fundamentalmente el soporte académico que ha de servir a la conducción de los procesos formativos entendidos como moldeamiento, mas no como ayuda a la constitución del ser, en el sentido en que positivamente lo reclama Gadamer (2005).

En cuanto a los responsables de la construcción del discurso pedagógico se consultaba en los ítems 6 y 7 . El primero hacía referencia a los profesores, el segundo a los pedagogos. Las valoraciones de estudiantes y profesores entregan valor importante tanto a unos como a otros. Es decir, tan relevante es el aporte al saber pedagógico de los maestros como el de los pedagogos. El ítem 9 confirma esta apreciación, ya que en este se consultaba si el saber pedagógico era solo asunto de maestros, ítem que tiene moderada valoración, posiblemente porque se reconoce por parte de los sujetos una importante aportación de otros actores. En igual dirección parece apuntar el ítem 8, que consultaba acerca de la relación entre experiencia y saber pedagógico. La relación de tal experiencia con los que la realizan en el aula posiblemente la asocian los consultados con el quehacer de los maestros, razón por la cual el acuerdo es moderado, por cuanto, en este ítem, otros que aportan a la construcción de tal saber no aparecen destacados.

Finalmente, en relación con las gráficas que son objeto por ahora de análisis, destacamos los ítems 10 y 11. El primero consultaba acerca del papel prevalente de la experiencia en la construcción del conocimiento pedagógico y el segundo, acerca de la prevalencia del saber del maestro en la construcción de tal conocimiento. Hay acuerdo, según se aprecia en las gráficas, en que tanto la experiencia como el saber disciplinar de los maestros ayudan a la constitución del conocimiento pedagógico.

Aunque no hay verdad definida en estos asuntos, es bueno colocar a consideración, a tono con los planteamientos de Cruz y Taborda (2014) la existencia de varias vertientes en pedagogía que intentan dar cuenta de cómo y quienes construyen saber pedagógico. Para algunos tal saber es del dominio de expertos, probablemente privilegiados, que se dan a la tarea de reflexionar sobre lo que debe ser la educación, en ocasiones alejados de la realidad de las instituciones educativas, planteamientos muy cercanos a lo que en términos de prescripción Ilama Benner (1991) educación afirmativa. La otra perspectiva es la que considera que el conocimiento pedagógico lo construyen aquellos que saben del acto de educar, en tanto reflexionan diariamente sobre este. Los autores en mención señalan, en 
tal sentido, que la pedagogía es teoría de la experiencia del educar, en un intento por sacar a la luz el papel importante de los maestros y de su experiencia comunitarizada.

Somos del criterio según el cual no se debe excluir de este análisis ninguna de estas versiones ni aquellas que dan cuenta de la pedagogía como "discurso que se ocupa del sentido de las prácticas educativas" o como "saber teórico teórico-práctico producto de la reflexión de los pedagogos en torno a su propia práctica" o como "discurso que conceptualiza, aplica y experimenta los conocimientos referentes a la enseñanza de los saberes específicos de las diferentes culturas", en la dirección en la que acertadamente lo desarrollan Sánchez, et ál. (2012, p. 45) al intentar constituir una conceptualización amplia del campo de la pedagogía.

\section{Comparación de categorías por licenciaturas: pedagogía}

Para una mejor comprensión del proceso investigativo se realizó un análisis comparativo de las ideas y respuestas que expresaron estudiantes de las licenciaturas, el cual se muestra en las siguientes figuras:

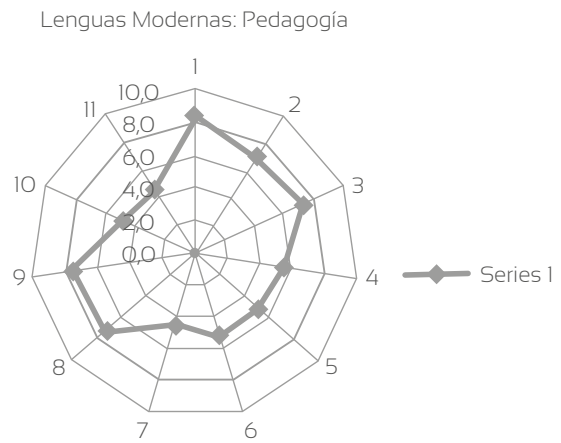

Figura 3. Valoración sobre pedagogía, estudiantes de Lenguas Modernas

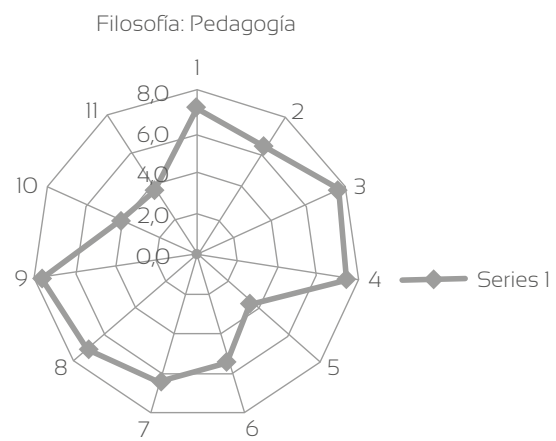

Figura 4. Valoración sobre pedagogía, estudiantes de filosofía 


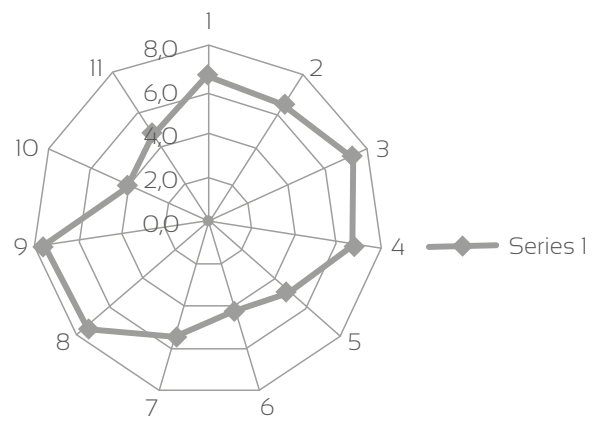

Figura 5. Valoración sobre pedagogía, estudiantes de Música Fuente: elaboración propia

Artes escénicas: Pedagogía

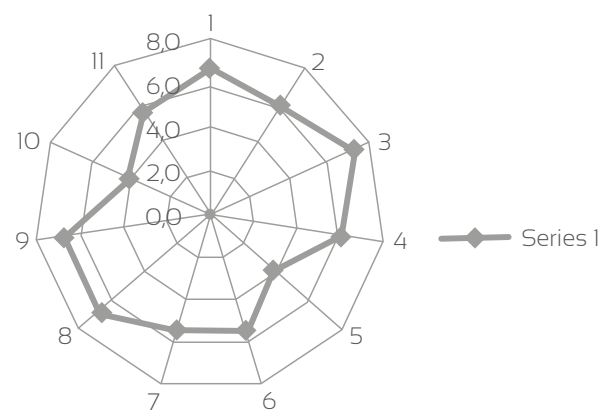

Figura 6. Valoración sobre pedagogía, estudiantes de Artes Escénicas Fuente: elaboración propia

Ciencias Sociales: Pedagogía

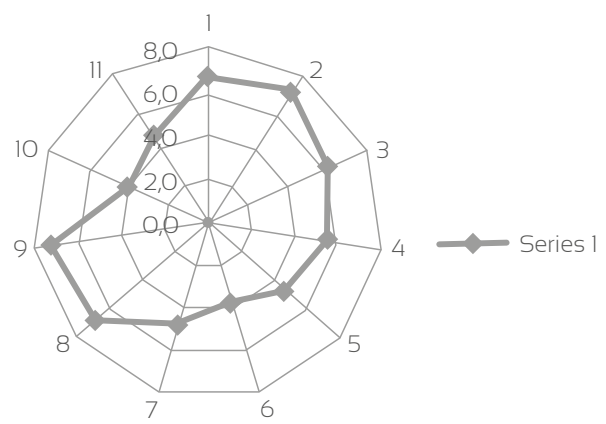

Figura 7. Valoración sobre pedagogía, estudiantes de Ciencias Sociales Fuente: elaboración propia 


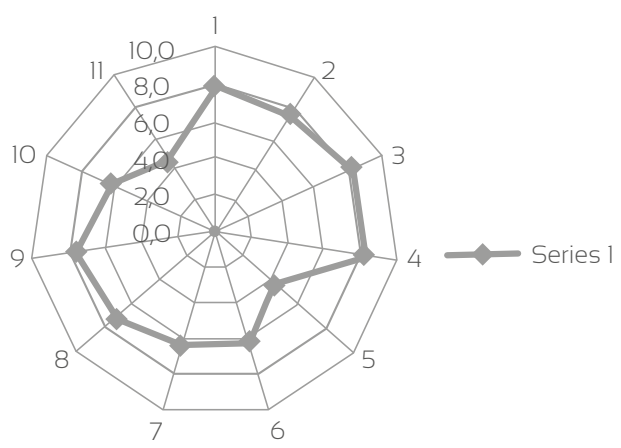

Figura 8. Valoración sobre pedagogía, estudiantes de Educación Física Fuente: elaboración propia.

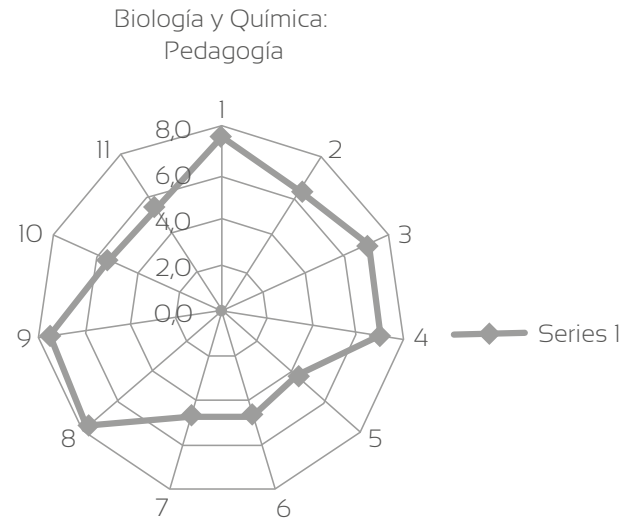

Figura 9. Valoración sobre pedagogía, estudiantes de Biología y Química Fuente: elaboración propia

Aparecen a continuación como objeto de análisis los datos de las figuras 3 a 9 de las licenciaturas en Lenguas Modernas, Filosofía, Música, Artes Escénicas, Ciencias Sociales, Educación Física, y Biología y Química, todos ellos de la Universidad de Caldas de Manizales.

La consulta acerca de si la pedagogía es un arte, alcanza en todos los programas académicos un alto nivel de acuerdo; en la Licenciatura en Lenguas Modernas la valoración es aún mayor. La asociación entre esta declaración de la pedagogía como un arte y el hacer, ya se ha justificado.

Adicionalmente podría decirse que primaría entre estudiantes, vinculada a la idea de la pedagogía como arte, en razón de su nivel de formación, una concepción tradicional del acto educativo que por su importancia en el pensamiento de los jóvenes los lleva a colocarle en íntima relación con la 
pedagogía, como si ese fuese su asunto fundamental no diferenciado de su objeto probable: la formación humana. Tal vínculo podría argumentarse en que en la pedagogía tradicional el arte de enseñar lo desarrolla el maestro. Tal pedagogía es caracterizada por Flórez (1999) asociando a ella como meta el humanismo y la formación del carácter y como métodos la transmisión, la imitación del buen ejemplo, el ejercicio y la repetición (p. 34).

A la formación humana, consultada en el ítem 2, en la mayoría de los programas se le ubica como objeto de la pedagogía con un alto nivel de acuerdo. En el programa de Biología y Química, la valoración es menor, aunque hay en promedio consenso en que es objeto de estudio de la pedagogía. Sería interesante, sin embargo, contrastar en otro informe estas expresiones en torno al objeto de estudio con resultados cualitativos aportados por nuestro instrumento de recolección de información, asunto que será tema importante en próxima publicación, por cuanto es posible que la formación asociada a la pedagogía tradicional se esté asumiendo como moldeamiento, muy cercano al interés de formar el carácter de la pedagogía tradicional.

En general como puede apreciarse en el ítem 3, la valoración es alta para afirmar que la pedagogía orienta el acto de enseñar en el aula. Esta afirmación relativa a si "es indispensable para el moldeamiento de los alumnos"; da a entender que todos los estudiantes están de acuerdo con que el saber pedagógico les permite dar soporte a las medidas que adoptan los maestros en formación sobre la autoridad que tienen para dirigir la formación del otro, más que para ser coadyuvantes en su constitución. En lenguas modernas, como puede apreciarse, la valoración para este ítem es más baja que en las otras licenciaturas. Sin embargo, en normalización, es decir, en la posibilidad manifiesta que pueden tener los docentes de que se respeten las normas y la disciplina en la escuela, en los programas de música, filosofía, educación física y artes escénicas se aprecian valores ligeramente menores que en los otros programas. De todas maneras, este ítem, como el anterior, asociados a la disciplina y el control, son consistentes en los estudiantes: tienden a estar de acuerdo los estudiantes en que la pedagogía aporta elementos para dar cuenta del moldeamiento y de la normalización de los estudiantes en las instituciones educativas.

Hasta el momento, entonces, aparecen importantes coincidencias en las respuestas de los estudiantes. En primer lugar, identificar la pedagogía con un arte se puede mirar como asociado a la pedagogía tradicional y en ella, con el moldeamiento como asunto importante en la formación en la cual el papel protagónico lo tiene el maestro. En segundo lugar, la pedagogía soporta lo que hace el maestro tanto en asuntos académicos como de normalización, es decir, de adaptación a las normas en la institución escolar. 
Cuando en los ítems 6 y 7 se pregunta por quiénes construyen saber pedagógico, los estudiantes de los programas de Filosofía, Música y Sociales dan más valor en este proceso de construcción a los pedagogos; en los otros programas, tanto profesores como pedagogos, en igual medida, aportan a la constitución de tal saber. Acá parece importante destacar que en el siguiente ítem, cuando se consulta acerca de la relación entre experiencia y pedagogía, las valoraciones de todos los estudiantes, en cuanto al nivel de acuerdo, son muy altas en todos los casos; es decir, lo que era leve diferencia entre programas en los ítems anteriores acá parece nivelarse a favor de los maestros, por cuanto su experiencia tiene gran relieve en la constitución de conocimiento pedagógico.

El ítem 9 consultaba si la pedagogía era asunto solo de maestros. En todos los programas se coincide en que además de los maestros otros actores desempeñan un rol importante; adicionalmente, a la experiencia de los maestros en la constitución de conocimiento pedagógico se le da baja valoración en los ítems 10 y 11 en unos programas y moderada en otros.

\section{Conclusiones}

Como parte del estudio "Semiosis de la pedagogía, el currículo y la didáctica", el presente artículo representa un avance importante en tanto las valoraciones desveladas del fenómeno "pedagogía" permiten proyectar relaciones importantes con el currículo y la didáctica. A continuación se presentan las principales conclusiones de este estudio, reportando de la pedagogía, en forma de enunciados generales, las principales valoraciones que sobre ella hicieron profesores y estudiantes de la Universidad de Caldas.

El estudio muestra que la mayoría de los estudiantes dan gran valor a la pedagogía como si fuese un arte. Tal valoración parece detener en el tiempo ideas consuetudinarias sobre el asunto y que advertían de la necesidad de considerar la vocación y el amor pedagógico como caracteres especiales que debía mostrar el educador. En la investigación al respecto, los maestros van más allá y asumen la pedagogía como conocimiento que se constituye a partir de otros conocimientos ya establecidos, y desde su propia experiencia reflexionada. Se esperaría que la enseñanza pudiese guiar a los maestros en formación a comprender la pedagogía en sentido más amplio y, en parte, integrando el pensamiento desvelado de los maestros sobre el tema.

Sobre la experiencia es necesario exponer que, tanto para profesores como para estudiantes, esta tiene un gran valor al momento de pensarla como determinante en la constitución de conocimiento pedagógico. Los docentes dan importancia similar al conocimiento constituido, esto relacionado posiblemente con su experticia y con sus estudios de maestría o doctorado,

o con titulaciones que tienen, razón por la cual le dan gran relevancia a

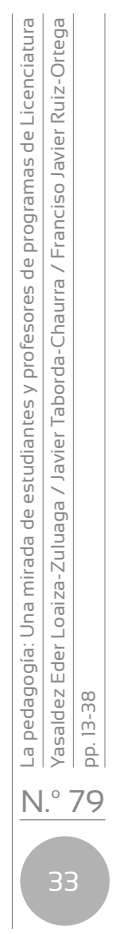


este conocimiento ${ }^{11}$. Lo dicho permitió mostrar que, en maestros y estudiantes, las ideas en torno a la experiencia están lejos de asociarse a esta como experimento o como directa relación de los sentidos con el mundo, y por lo cual, de tal suceso, simplemente se inscribiría en el entendimiento de los maestros, sin mediación de reflexión y como idea, aquello que viven en el aula. Es claro que para maestros y estudiantes la experiencia que aporta al saber de los maestros es experiencia reflexionada.

Ahora bien, como asunto de importancia, debe decirse que el reconocimiento que se hace de la formación como objeto de la pedagogía posiblemente le entregue a ella el papel de finalidad de la educación, razón por la cual es probable que haya identificación con el papel que cumple la pedagogía como campo en el cual el educar y el formar puedan estar en el centro de sus disquisiciones. Cabe mencionar que, en las expresiones de maestros y estudiantes consultados, el traslape de formación y educación o el asumir una u otra categoría como coordinada, supraordenada o subordinadas es cuestión común. En la literatura actual, igualmente, en diversos discursos tal traslape puede considerarse frecuente.

Otro asunto de interés, derivado del estudio, es que la pedagogía, reconocida en parte como reflexión del acto de educar o como teoría de la experiencia del educar, tiene respaldo en las valoraciones que dan estudiantes y profesores. El acto de educar y la enseñanza en las valoraciones se traslapa también ${ }^{12}$ y se le reconoce a la pedagogía el papel de dar soporte a lo que se desarrolla en la escuela. En este sentido, al parecer, para los estudiantes la pedagogía cobra importancia en la medida en que apoya los procesos de normalización en la escuela y en sus maestros; en una dimensión más amplia, amparada en su experiencia, es ella la que les permite colocar en segundo plano asuntos de control y centrar más atención en aspectos formativos.

Por último, tanto estudiantes como profesores reconocen un papel importante a los docentes quienes, con su experiencia y conocimiento, ayudan a la constitución de conocimiento pedagógico, al igual que a los pedagogos que sirven de referente a partir del saber que históricamente han venido construyendo.

11 Los maestros consultados pertenecen al Departamento de Estudios Educativos, unidad académica de la Universidad de Caldas que cuenta con uno de los más altos niveles de formación posgraduada en la institución a nivel de doctorados y maestrías.

$12 \mathrm{Al}$ igual que lo dicho para las categorías educación y formación. 


\section{Referencias}

Angulo, G. (2012). Las concepciones sobre el docente o diferentes maneras de concebir el ejercicio de la docencia. Revista de Investigación, 36(75), 11-32.

Benner, D. (1991) La capacidad formativa y la determinación del ser humano; sobre la pregunta y el planteo de una teoría de la formación no-afirmativa. Revista Educación, 43, 87-102.

Bollnow, O. (2001). Introducción a una filosofía del conocimiento. Buenos Aires: Amorrortú.

Bourdieu, P. y Passeron, J. (1970). La reproducción. Elementos para una teoría del sistema de enseñanza. Barcelona: Laia.

Bustamante, A. (2006). Educación, compromiso social y formación docente. Revista Iberoamericana de Educación, 37(4), 1-8. https://doi. org/10.35362/rie3742694

Carmona, Y. y Jacobs, A. (1975). Teacher Education, National Society for the Estudy of Education, 1974/1975. Bogotá: MEN-Icolpe-Programa Colombia-Unesco.

Castañeda, H. A. (2018). Arquitectura de aspectos sociales en la práctica pedagógica de profesores en formación de Lenguas extranjeras. Revista Colombiana de Educación, 75, 139-158. https://doi.org/10.17227/ rce.num75-8106

Cerleti, A. (2016). Repetición, novedad y sujeto en la educación. Un enfoque filosófico y político. Buenos Aires: Del Estante Editorial.

Cifali, M. (2005). Enfoque clínico, formación y escritura. En L. Paquay (Coord.), La formación profesional del maestro. Estrategias y competencias (pp. 170-196). México: Fondo de Cultura Económica.

Contreras, J. (2001). La autonomía del profesorado. Madrid: Morata.

Cruz, J. y Taborda, J. (2014). Hacia un giro fenomenológico en la pedagogía; el asunto de la experiencia en la pedagogía. Folios, 39, 161-171. https:// doi.org/10.17227/01234870.39folios161.171

Csikszentmihalyi, M. (2008). Fluir (Flow). Barcelona: Kairos.

De Tezanos, A. (1985). Maestros artesanos intelectuales. Estudio crítico sobre su formación. Bogotá: CIUP-UPN.

De Tezanos, A. (2006). El maestro y su formación, tras las huellas y los imaginarios. Bogotá: Cooperativa Editorial Magisterio.

Dewey, J. (2008). El arte como experiencia. Barcelona: Paidós.

Díaz, V. (2006). Formación docente, práctica pedagógica y saber pedagógico. Revista de Educación Laurus, 12 (número extraordinario), 88-103.

Durkheim, E. (1976). Educación como socialización. Salamanca: Ediciones Sígueme. 
Flórez, R. (1999). Evaluación pedagógica y cognición. Bogotá: McGraw-Hill. Foucault, M. (2005). Vigilar y castigar. México: Siglo xxı.

Freire, P. y Faundez, A. (2013). Por una pedagogía de la pregunta. Crítica a una educación basada en respuestas a preguntas inexistentes. Buenos Aires: Siglo XXI.

Gadamer, H. G. (2005). Verdad y método I. Salamanca: Sígueme.

García, R. (1986). Conceptos de "intención" y "educación": Análisis y estudio de relaciones. Revista Anales de Pedagogía, 4, 133-145.

Hegel, G. W. F. (2000). Escritos pedagógicos. México: Fondo de Cultura Económica.

Honoré, B. (1980). Para una teoría de la formación. Madrid: Narcea Ediciones.

Jay, M. (2003). La crisis de la experiencia en la era postsubjetiva. Santiago de Chile: Ediciones Universidad Diego Portales.

Kant, I. (1983). Pedagogía. Madrid: Akal.

Larrosa, J. (2006). Experiencia y narración. Revista Educación y Pedagogía, 18(separata). https://revistas.udea.edu.co/index.php/revistaeyp/article/view/19060/16283

Loaiza, A., Pinto, B., López, C., Fonseca, G., Aldaz, L. y Martínez, M. (2008). Urdimbre de sentidos de cultura escolar desde la multiculturalidad. Bogotá: Editorial Códice.

Loaiza, Y. (2018). Los maestros y la pedagogía. Revista Latinoamericana de Estudios Educativos, 14(1). http://190.15.17.25/latinoamericana/downloads/Latinoamericana14(1)_1.pdf

Loaiza, Y., Pineda, Y. y Arbeláez, N. (2014). Debate pedagógico: el maestro de las escuelas normales de manizales en el contexto del movimiento pedagógico. Manizales: Editorial Universidad de Caldas.

Ministerio de Educación Nacional (MEN) (2014). Sistema colombiano de formación de educadores y lineamientos de política: Bogotá.

Ocampo, J. (1999). La educación pública colombiana: 1950-2000. Revista Educación y Cultura, 40, 44- 58.

Organización de Estados Iberoamericanos (OEI) (2008). Cumbres y Memorias XVIII conferencia Iberoamericanas de Educación. El Salvador: Secretaría General Iberoamericana.

Parnett, J. (1986). La imagen del maestro en la historia. Revista Educación y Cultura, 8, 48-62.

Prats, J. y Raventós, F. (2005). Los sistemas educativos europeos. ¿Crisis o transformación? Barcelona: Fundación La Caixa.

Pozo, J., Scheuer, N., Pérez, M., Mateos,M., Martín, E. y De la Cruz, M. (Coords.) (2006). Nuevas formas de pensar la enseñanza y el aprendizaje, las concepciones de profesores y estudiantes. Barcelona: Graó. 
Rousseau, J. (2005). Emilio o de la educación. México: Editorial Porrúa.

Ruiz Ortega, F. J., Márquez, C., Badillo, E. y Rodas, J. M. (2018). Desarrollo de la mirada profesional sobre la argumentación científica en el aula de secundaria. Revista Complutense de Educación, 2(29), 559-576.

Sánchez, J., Ramírez, C., Chica, M. y Ávila, R. (2012), Coordenadas teóricas para la comprensión de las regiones investigativas en educación y pedagogía en Colombia. En H. Ospina y N. Murcia (Eds.), Regiones investigativas en educación y pedagogía en Colombia (pp. 33-76). Manizales: Editorial Zapata.

Schön, D. (1987). La formación de profesionales reflexivos, hacia un nuevo diseño de la enseñanza y el aprendizaje en los profesionales. Madrid: Centro de Publicaciones del MEC, Ediciones Paidós Ibérica.

Schiller, F. (1932). La educación estética del hombre en una serie de cartas. Madrid: Espasa Calpe.

Taborda, J. (2009). Sentidos de formación en maestros de una ENS colombiana. [Tesis de doctorado, Universidad de Caldas].

Trujillo, B. F. (2006). Educación cívica e intencionalidad educativa. Revista Internacional de Psicología y Educación, 8(1), 93-108. 